\title{
Current Leads for the LHC Magnet System
}

\author{
A. Ballarino
}

\begin{abstract}
The LHC current lead project is now entering into the stage of procurement of the components for integration into the machine. About 3300 current leads with ratings ranging from $60 \mathrm{~A}$ to $13000 \mathrm{~A}$ will be required for powering the numerous electrical circuits. The main design choices have been taken on the basis of the outcome of an intensive $R \& D$ program covering the characterization of suitable High Temperature Superconducting (HTS) materials for current leads, the validation of lead design options and the measurement of the thermo-electrical performance of prototypes tested under various operating conditions. Helium gas cooled leads integrating HTS material at the colder end will be used for high current leads (currents $\geq 600 \mathrm{~A}$ ), while the corrector dipole magnets (60 A-120 A) will be powered via specifically designed conduction-cooled resistive leads. An outline of the retained designs will be presented, together with required schedule for lead manufacture.
\end{abstract}

Index Terms-Accelerator technology, current leads, cryogenics, high temperature superconductors.

\section{INTRODUCTION}

$\mathbf{P}$ ARTICLE colliders, in which two beams circulating in opposite directions are made to intersect, seek to maximize the particle energy in the center of mass frame. In the Large Hadron Collider, $7 \mathrm{TeV}$ proton beams are curved to follow the LEP circumference by means of high magnetic bending fields $(8.3 \mathrm{~T})$ created by a system of superconducting dipoles. In addition to the dipoles, also quadrupoles, corrector dipole, sextupole, octupole and decapole magnets will be installed to focus and correct the particle beams. About 3300 current leads, rated for currents from $60 \mathrm{~A}$ to $13000 \mathrm{~A}$, will power this complex magnet system, providing the electrical link between the warm (room temperature cables) and cold (superconducting bus bars) electrical circuits.

Since High Temperature Superconductors (HTS) became available on an industrial scale, an important effort has been made at CERN to prove the feasibility of efficiently and safely incorporating these new oxide materials in current lead applications. On the basis of the positive outcome of an extensive R\&D program [1], covering both the characterization of different HTS materials and the measurement of several prototypes, tested under various operating conditions, CERN has decided to adopt the HTS technology for all the leads operating at currents ranging from $600 \mathrm{~A}$ to $13000 \mathrm{~A}$. The dipole corrector magnets, which represent about $5 \%$ of the total current, will be powered via specially designed resistive current leads.

While encouraged by the results obtained elsewhere, on HTS leads tested as prototypes [2] or already integrated into a real

Manuscript received September 24, 2001.

The author is with CERN, European Organization for Nuclear Research, CH-1211 Geneve 23, Switzerland (e-mail: Amalia.Ballarino@cern.ch).

Publisher Item Identifier S 1051-8223(02)04178-7. operating system [3], the LHC will be the first to incorporate a large number of HTS leads in a large scale accelerator system. An important effort has therefore been put into converging, for the series production, on a lead design able to guarantee good thermo-electrical performance together with characteristics of compactness, robustness and safety of the components.

\section{POWERING: NUMBER, RATING AND LOCATION OF LEADS}

Power converters located close to the magnets in underground caverns and service tunnels (with the exception of the $60 \mathrm{~A}$ orbit corrector converters which are in the main arc tunnel) feed about 1700 electrical circuits.

The powering of the main dipole magnets will be made via 8 electrical circuits, fed right and left of each of the even points, and including 16 energy extraction systems. The main quadrupole magnets will be grouped into 16 electrical circuits. Sixty-four HTS current leads, operating at up to 13000 A, are therefore required for the powering of the main magnets. The quadrupole and dipole magnets of the insertion regions, operating at currents ranging from $3900 \mathrm{~A}$ to $6900 \mathrm{~A}$, will require about 310 HTS current leads. An additional 750 HTS current leads will feed the $600 \mathrm{~A}$ corrector magnets. All these leads will be grouped in specially designed cryostats, called Distribution Feed Boxes (DFB), located right and left of each of the 8 interaction points.

The 60 A corrector dipole current leads, 1504 in total, will be located in the arcs and will be directly integrated in the quadrupole cryostat technical service modules.

The 120 A corrector dipole current leads, 504 in total, will be located in the Dispersion Suppressor regions and in the Matching Sections. The present machine layout groups some of them in the DFB and some in the quadrupole cryostat technical service modules.

These leads will transport all together more than 3.4 MA of current.

\section{HTS LEADS}

The HTS current leads project at CERN has evolved through different stages: optimization of the thermo-electrical performance of the leads within the defined infrastructure of the LHC machine, tests of HTS elements, elaboration of lead conceptual design, issue of functional specifications for prototypes which were built in industry and characterized at CERN and design of leads for the series production.

The experience gained with the prototypes, which includes extensive measurements of several $13000 \mathrm{~A}$ and $600 \mathrm{~A}$ HTS leads [1], has been an important step in the evolution toward the final design. It has allowed not only to confirm 
design parameters (cooling scheme, operational temperature of HTS, cryogenic and electrical performance, assessment of geometrical constraints) and to validate theoretical calculations (thermo-electrical model of resistive and HTS parts), but also to identify the critical parts in the engineering design of the lead. The most common problems encountered during the prototype tests were:

- high resistance joints, localized either at the cold end of the resistive part or at the warm or cold end of the HTS, preventing the lead from reaching the nominal current (premature quench of HTS) or spoiling the thermal performance (overcooling of resistive part or high heat load into the helium bath),

- leak tightness problems in different parts of the lead, in particular at the interconnection between the resistive and the HTS part, making the hydraulic separation between the $20 \mathrm{~K}$ and the $4.5 \mathrm{~K}$ helium circuits, and in "plugs" integrated in the lead to assure both electrical insulation and leak tightness,

- incorrect dimensioning of the resistive heat exchanger with consequent need of $20 \mathrm{~K}$ helium gas overflow.

The design of the leads for the machine is based on a single resistive heat exchanger unit, extending from the top of the lead down to the connection of the HTS. Intermediate electrical joints are avoided by including in the machining of the fin-type heat exchanger two copper ends acting one as support of the warm electrical terminal and one as warm end-cap of the HTS. The HTS element consists of an assembly of stacks of BSCCO 2223 tapes. Electrical and mechanical interfaces (warm cables, connection of HTS to low temperature superconducting bus bar and warm flange supporting the leads onto the cryostat flange) are optimized for integration into the final DFB.

\section{A. 13000 A Leads}

The lead consists of a live envelope, which includes the warm electrical terminal, the resistive heat exchanger with a vacuum insulation jacket and the HTS element. A warm insulating flange provides the electrical insulation of the lead body from the cryostat. The conductor is a copper rod (RRR $\approx 120$ ) with fins machined from the main copper body. These fins are thin plates cut by turning to allow the helium gas to flow, between two fins, in a zig-zag fashion. The conductor length and cross-section are optimized to carry 13000 A with a temperature at its cold end of about $50 \mathrm{~K}$. The fins thickness and radial extension are optimized for heat transfer between the conductor and the gas. Operating in a range of temperature above $50 \mathrm{~K}$, the heat exchanger performance is not as much influenced by the properties of the copper alloy as it is that of a conventional self-cooled lead. This means that variations of RRR during the assembly procedure (machining and brazing) are less important. Low resistivity copper is preferred at the ends for the electrical connections. Making the heat exchanger as a single unit, the use of copper with $\mathrm{RRR} \approx 120$ guarantees low resistance joints, good thermal performance and compact design. A vacuum insulation jacket, extending all along the resistive heat exchanger, assures the thermal insulation of the
$20 \mathrm{~K}$ helium circuit from the cryostat environment (helium gas ( 1.3 bar). An insulating tube encases the lead body inside the cryostat. A $20 \mathrm{~K}$ helium mass flow of about $0.7 \mathrm{~g} / \mathrm{s}$ is required for operating the lead at nominal current. This flow is regulated as a function of the temperature of the top end of the HTS part [1].

A copper block is brazed around the warm end of the heat exchanger. It includes the recovery $20 \mathrm{~K}$ helium circuit and the copper plate for the connection of the warm cables. Heater cartridges are integrated at the top of the lead to maintain the room temperature in stand-by and low current operation. The electrical connection, parallel to the lead axis, does not extend past the flange diameter, which is about $0.2 \mathrm{~m}$. This design allows for the integration, from the top of the lead, of a stainless steel chimney, which is part of the DFB cryostat.

The HTS element consists of a stainless steel tube on which are disposed stacks of BSCCO $2223 \mathrm{Ag}-\mathrm{Au}$ tapes. The number of tapes per stack can be adapted according to their current density. The cross section of the stack is constant along the length. The stacks are distributed around the stainless steel cylinder in a configuration that minimizes the magnetic field acting in the direction perpendicular to the tapes. Tests at CERN have shown that satisfactory stacks can be made by $\mathrm{SnAg}$ soldering tapes together in a vacuum furnace using a suitable jig.

The cross section of the $\mathrm{Ag}-\mathrm{Au}$ alloy is calculated to satisfy the quench protection requirements of the most critical $13000 \mathrm{~A}$ circuit. In case of resistive transition of the high temperature superconductor, the lead should be able to discharge the magnet chain at its nominal current decay rate, corresponding to a time constant of about $120 \mathrm{~s}$, without overheating above $200 \mathrm{~K}$.

Gas vaporized at $4.5 \mathrm{~K}$ by the conduction of the HTS element is guided to cool the HTS by a surrounding fiber-glass cylinder. This gas escapes at the top of the HTS into the cryostat environment. The heat load into the liquid helium at nominal current is of the order of $1.5 \mathrm{~W}$ [1].

At the cold end of the HTS low temperature superconducting NbTi wires (two per stack), are soldered in grooves included in the copper end-cap. These wires are part of two Rutherford cables and assure a flexible joint. One of their ends is prepared for the connection to the bus bar. The copper stabilizer required for the protection of the bus bar (about $280 \mathrm{~mm}^{2}$ ) consists of flexible copper sheets. It is part of the bus bar supply and it is bolted from the bottom onto the copper end-cap. Two thin copper sleeves are soldered around the HTS stacks along the length that will be covered by liquid helium.

Leak tightness between the $20 \mathrm{~K}$ and the $4.5 \mathrm{~K}$ circuits, at the interconnection between the resistive part and the HTS element, is guaranteed by the welding of the vacuum jacket onto a stainless steel insert brazed at the lower end of the heat exchanger.

The instrumentation integrated into the leads includes two temperature sensors at the top of the HTS, used for the control of the $20 \mathrm{~K}$ helium flow, and two voltage taps, at the top and at the bottom of the HTS, for quench protection of the superconductor. The instrumentation wires for these signals, which belong to the $4.5 \mathrm{~K}$ helium circuit, are brought out through a tube in the resistive heat exchanger. Leak tightness between the $4.5 \mathrm{~K}$ circuit and the outside environment is made at warm, at the top 


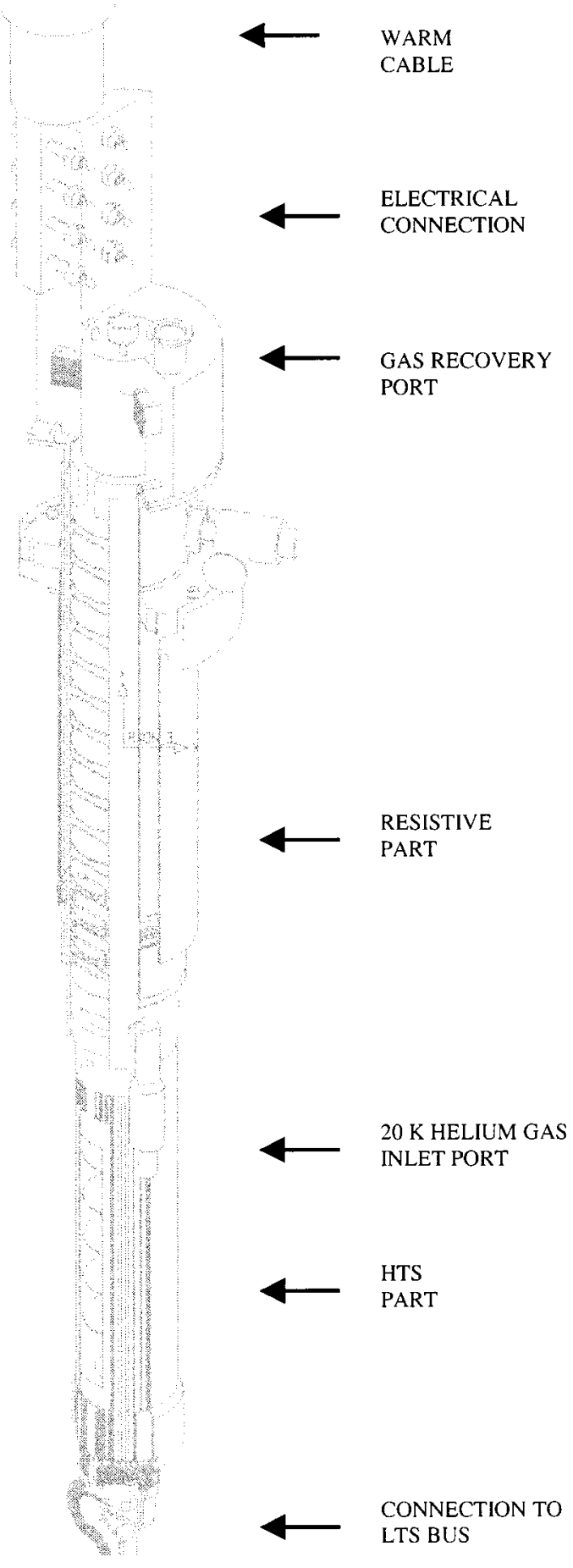

Fig. 1. 13000 A HTS current lead.

of the resistive heat exchanger, where a connector is mounted onto a stainless steel insert brazed in the copper terminal. One additional voltage tap is integrated at the top of the resistive heat exchanger for protection in case of thermal run-away.

The total length of the lead, including the warm electrical flag, is about $1.45 \mathrm{~m}$. It has been reduced to the minimum to allow integration and transport of the equipment in the LHC tunnel. The external diameter of the lead body is about $90 \mathrm{~mm}$. The leads have been designed to be as compact as possible to reduce to the minimum the pitch at which they can be integrated in the cryostat.

\section{B. 6000 A Leads}

The design of the $6000 \mathrm{~A}$ leads powering the magnets of the insertion region is similar to that of the $13000 \mathrm{~A}$ leads. These leads will operate at currents ranging from $3900 \mathrm{~A}$ to $6900 \mathrm{~A}$. In order to have one single design covering the whole range of current, the geometry of the lead is optimized for a value of about $6000 \mathrm{~A}$. To maintain optimum performance at lower or higher ratings, the regulation of the $20 \mathrm{~K}$ helium flow is adapted by appropriate choice of the set temperature, different from the nominal $50 \mathrm{~K}$, at the top of the HTS. In view of the large number of these leads, emphasis has been put on a compact design. The cold connection of the HTS element to the low temperature superconductor is simpler than that of the $13000 \mathrm{~A}$ leads in view of the smaller amount of copper required as stabilizer for the bus bar (about $4 \mathrm{~mm}^{2}$ ). The low temperature superconductor (LTS) consists of a standard 36 strands cable of NbTi.

\section{600 A Leads}

The $600 \mathrm{~A}$ leads are assembled in groups of four on a common insulating flange. Their design is similar to the high current leads. The assembly of four leads has been made as compact as possible. The external diameter of the common mounting flange is about $0.26 \mathrm{~m}$. The total length of the lead is about $1.2 \mathrm{~m}$. A common manifold, providing electrical insulation, distributes the $20 \mathrm{~K}$ helium gas in each lead.

The $20 \mathrm{~K}$ mass flow required for operating the resistive heat exchanger at nominal current is about $30 \mathrm{mg} / \mathrm{s}$. The correspondent heat load into the helium bath is about $0.07 \mathrm{~W} /$ lead [1].

Low temperature NbTi superconducting wires, soldered at the cold end of the HTS, provides the electrical link to the superconducting bus bar.

\section{RESISTIVE LEADS}

The $60 \mathrm{~A}$ and $120 \mathrm{~A}$ current leads do not integrate HTS material. Besides the fact that the gain in heat load would be low as compared to the high current leads and that they represent only a small percentage of the total current, their integration in the final cryostat is such that the use of HTS material would not be appropriate. The $60 \mathrm{~A}$ leads are the last components integrated in the quadrupole cryostat technical service modules (QQS): their shape has to be adapted to fit in the space available.

\section{A. 60 A Leads}

The 60 A leads are conduction cooled. Their design is based on requirements of simplicity (no active flow is foreseen for cooling the element, to avoid control valves and piping for the recovery of the lead gas return), mechanical flexibility for easy integration, and low heat load into the liquid helium.

The lead consists of a pure conduction cooled wire operating between room temperature and the $1.9 \mathrm{~K}$ liquid helium bath. To minimize the heat dissipated into the liquid, the conductor is thermalized at two intermediate temperature levels. A first heat sink is provided by the $50 \mathrm{~K}$ to $75 \mathrm{~K} \mathrm{He}$ gas of the line $\mathrm{E}$, cooling 

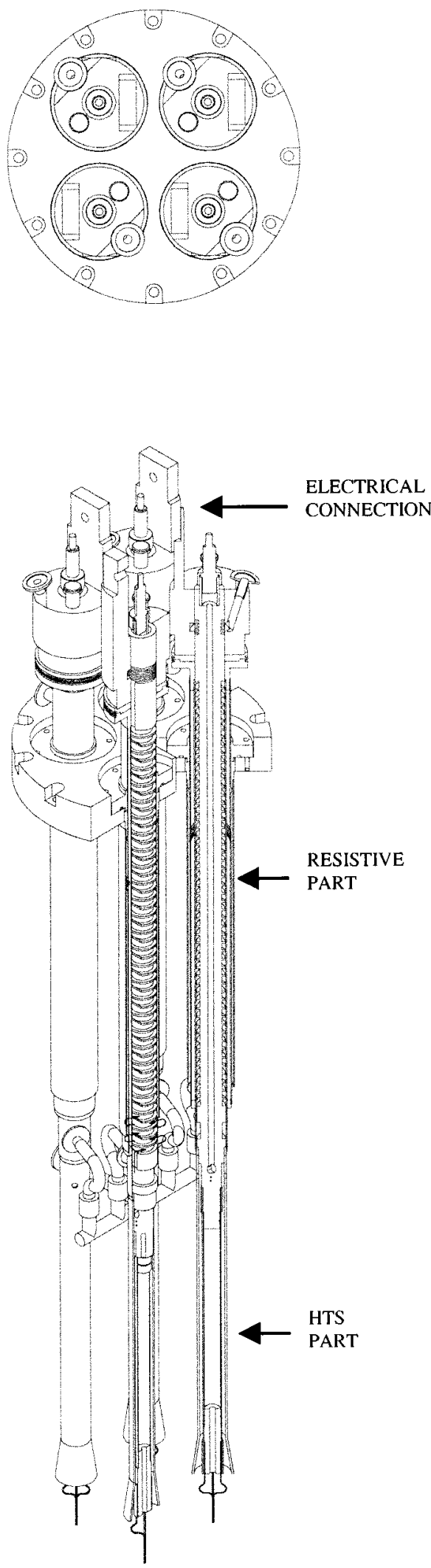

Fig. 2. Assembly of four 600 A HTS current leads.

the thermal screen, and a second heat sink is provided by the 20 $\mathrm{K}$ He gas of the line $\mathrm{C}^{\prime}$, cooling the beam screen.

The conductor, electrically insulated by a Kapton ${ }^{\circledR}$ tube, is contained in a thin stainless steel tube welded at the two ends onto stainless steel flanges. The leak tightness with respect to the vacuum environment through which the lead passes is guaranteed by the welding of the flange onto the cold mass, at the cold end, and at the main cryostat flange at the warm end. The insulated conductor fills more than $90 \%$ of the cross section of the tube; elsewhere the helium gas from the $1.9 \mathrm{~K}$ bath stratifies inside the stainless steel tube. The leak tightness of the $1.9 \mathrm{~K}$ circuit with respect to the outside environment is guaranteed, at the top of the lead, by a warm o-ring.

Each assembly consists of four leads mounted onto a common flange. The leads are pre-shaped as required for the integration in the QQS and mounted from the end of the cryostat. The thermalization of the heat sinks is made by clamping the stainless steel tubes onto plates integrated onto the line $\mathrm{E}$ and $\mathrm{C}^{\prime}$.

Several alloys have been studied for the optimization of the conductor. In order to minimize the heat load at the heat sinks and helium bath and in view of the fact that the length of the lead is imposed by the space available in the cryostat, the optimum thermal performance is obtained by varying the conductor cross section along the length. The material should also have a small cross section in order to be easily pre-shaped and should conduct a minimum of heat in stand-by and low current operation, since the corrector magnets are rarely operated at maximum current. In addition, as the tapered conductor is housed in a stainless steel tube with a constant cross section, the difference in the conductor diameter should be small in order to allow clamping and efficient thermalization. Changes in stainless steel cross section (drawn tube) would make the design more complex and more expensive.

All these requirements have been met by selecting as electrical conductor a brass wire with two different copper electrolytic depositions: a thicker one, about $900 \mu \mathrm{m}$, over the upper part, including the $50 \mathrm{~K}$ to $75 \mathrm{~K}$ thermalization, and a thinner one, about $110 \mu \mathrm{m}$, over the lower part, including the $20 \mathrm{~K}$ thermalization. Since this "hybrid" conductor does not follow the Wiedemann-Franz-Lorentz (WFL) law, a gain in thermal performance is obtained with respect to a WFL material (about $10 \%$ @ $50 \mathrm{~K}-75 \mathrm{~K}, 25 \% @ 50 \mathrm{~K}$ and $8 \%$ @ $1.9 \mathrm{~K}$ ). This is important in view of the large number of leads.

A critical part of the lead design is the thermalization of the heat exchangers. The clamping plates have grooves into which the stainless steel tubes are squeezed to get in contact, through the Kapton ${ }^{\circledR}$ insulation, with the "hybrid" conductor. Care has been taken to maintain the required clamping force after cooldown. Protection of the leads is required in case of loss of thermalization and consequent thermal run-away of the conductor.

\section{B. 120 A Leads}

The conduction cooled 120 A leads are of the same design of the resistive $60 \mathrm{~A}$ current leads (same length and adapted cross section). The 120 A leads integrated in the DFB are conventional self-cooled leads.

\section{STRING 2 EXPERIENCE}

Six 13000 A and twenty-six 600 A HTS prototype leads, already tested in a dedicated cryostat, have been mounted in the String 2, a full size model of the LHC cell. This has allowed 


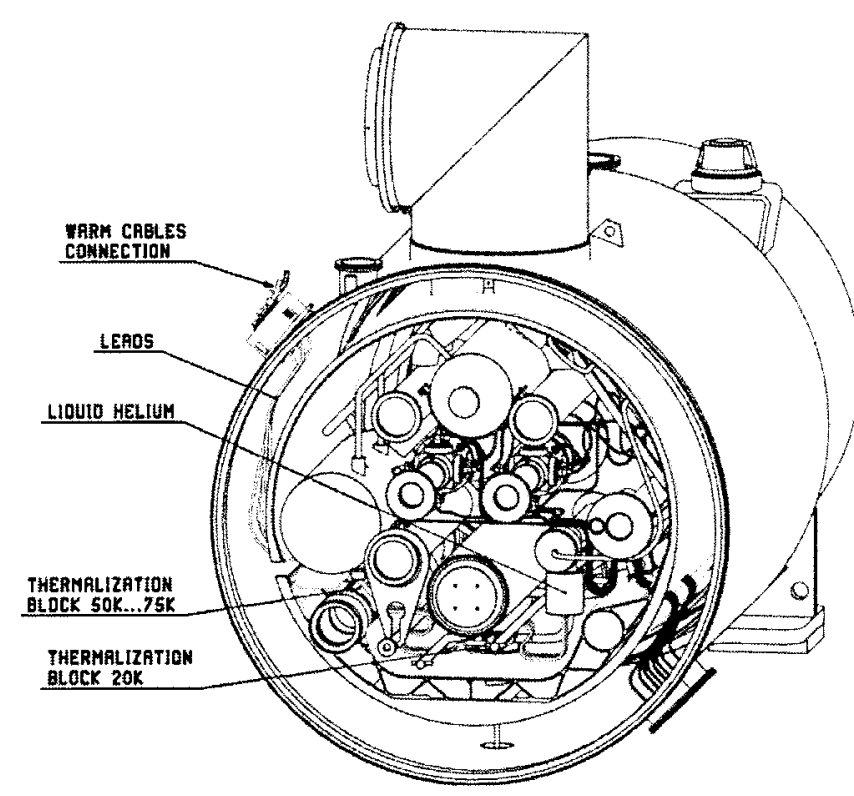

Fig. 3. Assembly of four $60 \mathrm{~A}$ leads integrated in the quadrupole cryostat technical service module.

to gain experience with the integration of the components in a complete system where the leads, confined in a limited space between the two beam pipes, have to satisfy constraints given by several interfaces (mechanical, cryogenic and electrical). Electrical interconnection between the leads and the bus bar have been designed and manufactured for this special application. Their installation has been made through the openings foreseen from the side of the cryostat.

The String 2 is now cold and being commissioned. Its operation will provide the opportunity of acquiring additional experience with long term operation and thermal and electrical cycling of the leads.

Two assemblies of four $60 \mathrm{~A}$ current leads have been integrated in the String 2. This has been an important stage for the confirmation of the design parameters, in particular of the geometrical constraints given by the cryostat assembly. Prototype leads have been separately tested in a dedicated cryostat.

\section{SCHEDULE FOR LEADS MANUfACTURE}

The delivery of the leads will have to be in line with the schedule foreseen for the installation of the LHC machine. According to the present plan, the first DFB equipped with current leads will be installed in the LHC tunnel in January 2004 (DFB left of point 8 ). The first HTS series units must be available early in 2003. The thermo-electrical behavior of the pre-series leads will be characterized in a dedicated cryostat. All HTS current leads will be cold tested at nominal current, once integrated in the final cryostats, prior being installed in the LHC tunnel. Integration in the machine should be completed by the end of 2005 .

The finalization of the design for the $600 \mathrm{~A}, 6000 \mathrm{~A}$ and 13000 A HTS current leads is very well advanced. Prototype work, including the test of specific features of the leads, is being carried out in parallel with the detailed design work. Three technical specifications for the supply of leads rated for the three different current ratings, each including a CERN HTS lead ref-

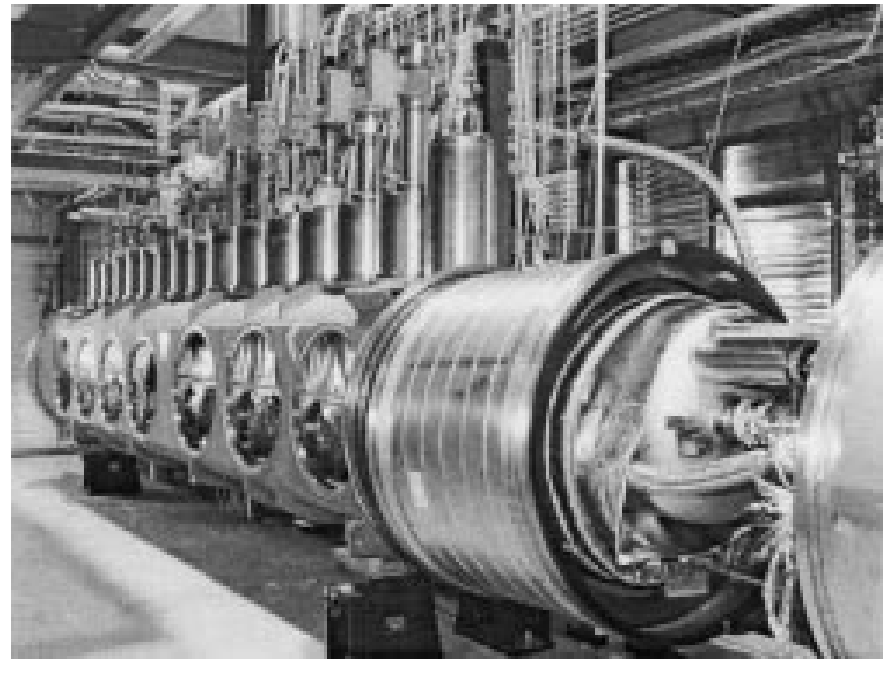

Fig. 4. Prototype HTS leads integrated in the String 2.

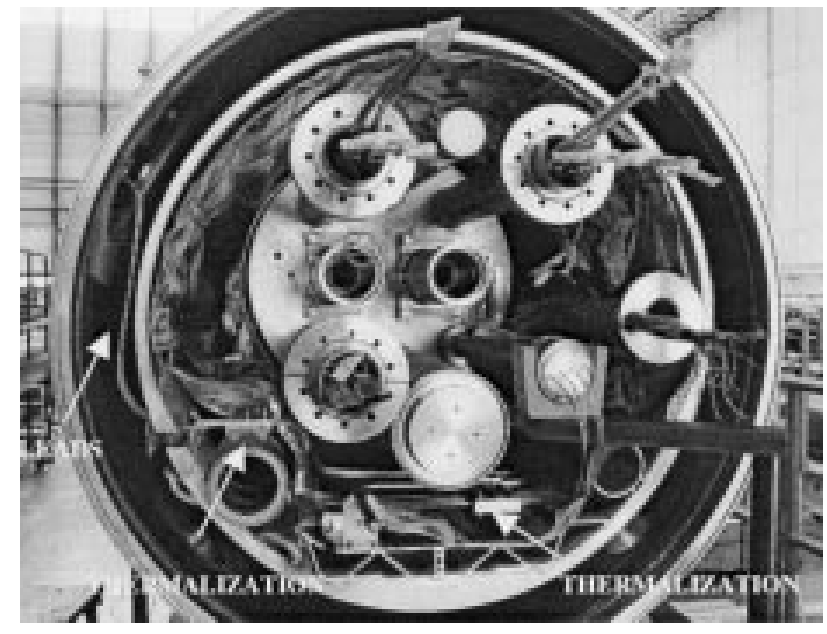

Fig. 5. Prototype 60 A leads integrated in SSS3.

erence design, will be issued starting from the end of 2001. The tendering phase should be completed by the middle of 2002 .

The 60 A resistive current leads assemblies should be ready for integration as from January 2002. The first 60 A series units are being prepared at CERN. A technical specification based on the CERN design for the series production of the $60 \mathrm{~A}$ and $120 \mathrm{~A}$ resistive current leads will be issued in the near future.

\section{CONCLUSION}

The design and development stage of the current leads for the LHC machine is moving toward completion, and we are now entering into the stage of procurement of the components for integration into the machine.

The feasibility of using HTS material for high current leads, with important savings in cooling power [4], has been proved with the work performed on the prototypes [1]. A reduction of heat load into the helium bath of a factor greater than 10 if compared to conventional self-cooled leads has been measured on several $13000 \mathrm{~A}$ and $600 \mathrm{~A}$ HTS prototype leads [1]. The corresponding reduction in overall cooling power is about a factor of 3 . 
The design of the leads for the machine is very well advanced and the tendering phase will start as from the end of 2001.

The resistive leads for the corrector magnets have been specifically designed and their production will start at the end of 2001.

\section{REFERENCES}

[1] A. Ballarino, "HTS current leads for the LHC magnet powering system," in EUCAS Proc., 2001.
[2] R. Heller, K. Takahata, G. Friesinger, T. Mito, M. Tasca, A. Nishimura, S. Satoh, and S. Yamada, "Test results of $20 \mathrm{kA}$ current leads using Ag/Au stabilized Bi-2223 tapes," IEEE Trans. Appl. Superconduct., vol. 11, pp. 2603-2606, Mar. 2001.

[3] G. Citver, E. Barzi, A. Burov, S. Feher, P. J. Limon, and T. Peterson, "Steady state and transient current lead analysis," EEE Trans. Appl. Superconduct., vol. 9, pp. 515-518, June 1999.

[4] A. Ballarino, "Application of high temperature superconductors to accelerators," in EPAC Proc., 2000, pp. 227-231. 